\title{
A NEW X-RAY DIFFRACTION APPARATUS
}

BY

WheELer P. DAVEy

The use of monochromatic X-Rays for the determination of crystal structure is well known. It is the purpose of this article to describe an apparatus by which the characteristic X-Ray diffraction patterns of fifteen powdered crystals may be taken at once. Each pattern is recorded on a strip of photographic film $17 / 8 \times 16$ inches, placed in a cassette which automatically holds the film on the arc of a circle of 8 inch radius, having at its center the glass tube containing the specimen of powdered crystal. These cassettes are placed radially on a circular horizontal table at a fixed distance from a vertical water-cooled Mo-target Coolidge tube. The fifteen specimens thus lie along the edges of a horizontal polygon whose center is on the axis of the Coolidge tubes. The Coolidge tube is excited by a transformer placed under the table. In this way, the whole apparatus, exclusive of the switchboard, occupies a space 3 feet in diameter and about $41 / 2$ feet high (see Fig. 1).

The time required to obtain a satisfactory record of the diffraction pattern varies from 5 to 12 hours for substances like $\mathrm{NaCl}$ to 48 to 70 hours for substances like $\mathrm{CuCl}$ and $\mathrm{CsI}$. The diffraction pattern may be interpreted directly in terms of the interplaner distances in the crystal by means of a metal scale which is calibrated in Ångstrom units. If the crystal happens to belong to the cubic, tetragonal, or hexagonal systems its structure may be interpreted directly from these interplaner distances by means of graphs described below.

\section{The TABLE}

The table-top consists of a disk of steel $1 / 2$ inch thick, provided with 15 radial guides for the cassettes. A hole 12 23/64 inches in diameter is cut in the center. In this hole is fitted a steel cylinder $119 / 16$ inches high which acts as a support for the slit system, and at the same time, because of the long wave length of X-Rays 
employed, provides $\mathrm{X}$-Ray protection for the investigator. Fifteen rectangular openings at the level of the slit system allow the rays to reach the glass tubes containing the specimens. Under each of these openings is another opening which may be used. when desired for calibrating the photographic film. It is, of course, imperative, that all openings not covered by cassettes be blocked off by sheet lead or by $1 / 8$ inch sheet brass to avoid danger to the investigator. The lead or brass should overlap the holes by at least half an inch.

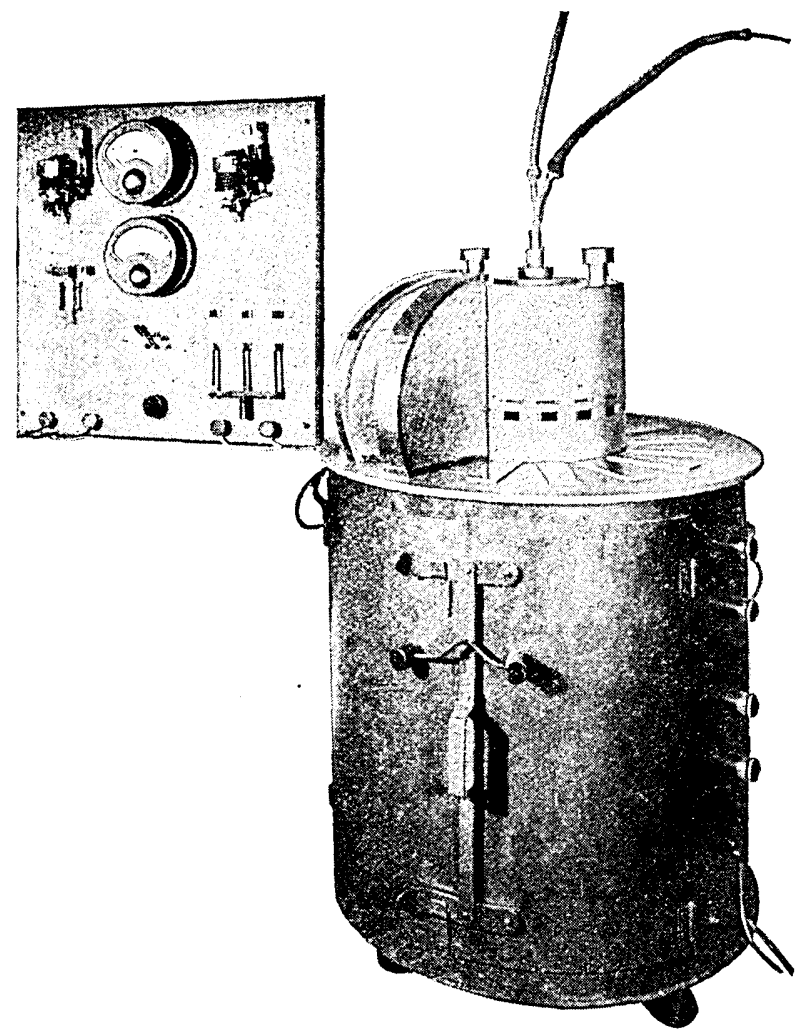

Fig. 1A-X-Ray Diffraction apparatus (doors closed)

The top of the cylinder is closed by a flat brass cover which also acts as a rigid support for the anode end of the Coolidge tube. The cathode end of the tube hangs freely. The cylinder and cover are machined so that the axis of the tube automatically 
coincides with the axis of the cylinder. A set-screw allows a vertical adjustment of the tube along the axis of the cylinder.

The under part of the table is enclosed by a cylinder of heavy sheet ịron, reënforced with angle iron, and provided with doors to give access to the high-tension transformer which sits inside (see Fig. $1 \mathrm{~B}$ ). The primary circuit of the transformer is carried

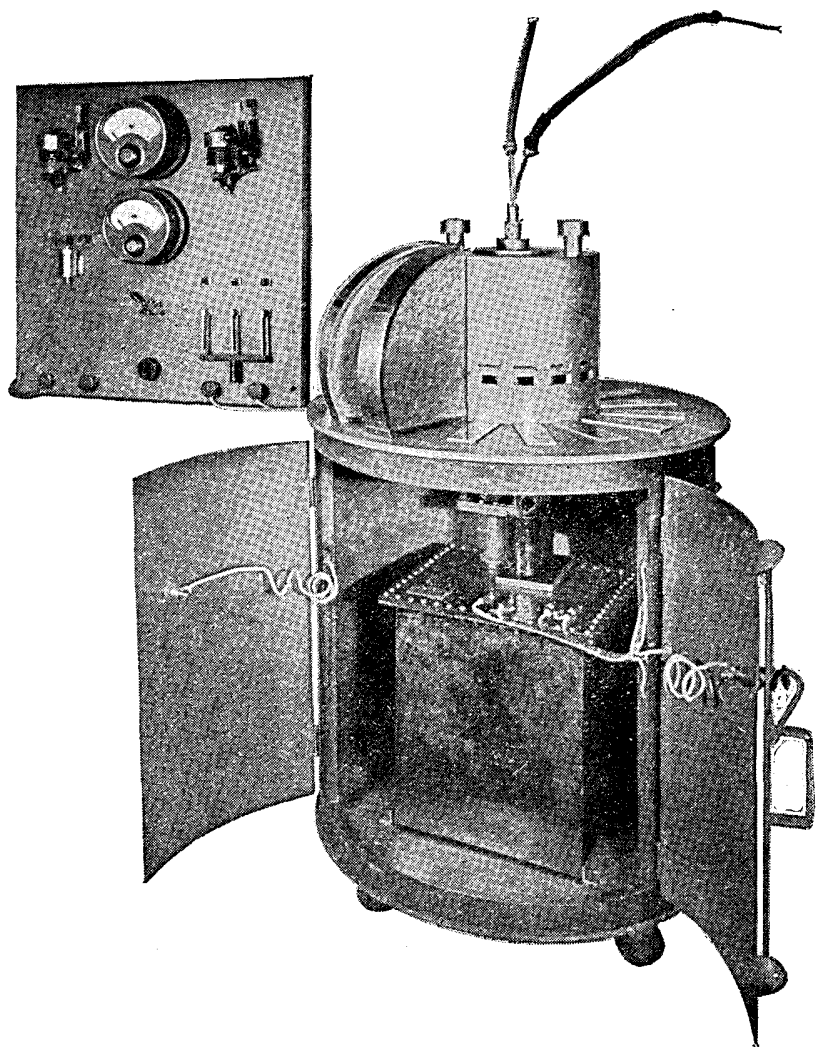

Fig..1B. X-Ray Diffraction apparatus (doors open)

across the front of these doors in such a way that the doors can not be opened without opening the primary circuit (see Fig. 1A). In this way, when the X-Ray tube is in operation, the operator is protected from accidental contact with the high potential.

The whole table is mounted on heavy castors so that it may be easily moved from place to place. 


\section{The Tube and Its Accessories}

The X-Ray tube is of the usual Coolidge type, but with a special water-cooled anode. This anode consists of a hollow copper rod at the end of which is fastened a molybdenum button. The shape of the rod is such as to provide an easy heat-path from the button to a large surface of water, so that local boiling is avoided. The face of the Mo button is perpendicular to the axis of the tube, so that X-rays may be taken off all the way around the anode.

The cathode is the standard 30 ma. cathode commonly found in the Radiator Type Coolidge tubes supplied to roentgenologists. Because of the large anode-cathode distance and the low voltage employed ( $30 \mathrm{kv}_{\mathrm{R} \text { s }}$ ) the tube shows space-charge characteristics, that is, the voltage drop immediately in front of the cathode filament is so low that when the filament operates at normal heating current (say 4.5-4.75 amperes), fewer electrons are dragged from the cathode to the anode than are evaporated from the filament. For this reason, at a definite voltage across the tube the current is independent of the filament temperature provided that the temperature exceeds a certain minimal value. A tube operated so as to show space-charge characteristics appears to be more efficient in crystal analysis work than one having the characteristics of the ordinary Coolidge tube, in which the space-charge characteristics are noticeable at lower voltages, but in which a higher percentage of the electrons is utilized at $30 \mathrm{kv}$. There is, besides, this additional advantage, that the tube load is nearly constant even though the filament is heated from a variable source of potential, provided that the variation in voltage on the filament circuit is less than $5 \%$.

The maximum allowable filament current for 1000 hours life is 4.75 amperes. The maximum tube voltage for efficient production of Mo characteristic rays is $30 \mathrm{kv}$ Rass. At this voltage the tube current should be about $30 \mathrm{ma}$, but will vary from 28 to $35 \mathrm{ma}$ from one tube to another.

Dimensions of the tube are shown in Fig. 2.

The radiation from the tube is filtered through two filters of $\mathrm{ZrO}_{2}$, each having $.05 \mathrm{~g}$. of $\mathrm{ZrO}_{2}$ per $\mathrm{cm}^{2}$. One filter is built into 
the slit system,- the other into the cassettes. The first filter eliminates most of the "white" (general) radiation, and a large fraction of the Mo $\beta$ doublet $(.63 \AA)$. The second filter makes the rest of the Mo $\beta$ doublet negligible, and eliminates a large part of such characteristic rays as may be given off by the specimen itself. The diffraction pattern, as recorded on the photographic film, is therefore caused by the Mo a doublet (average wave length .712 $\AA$ ).

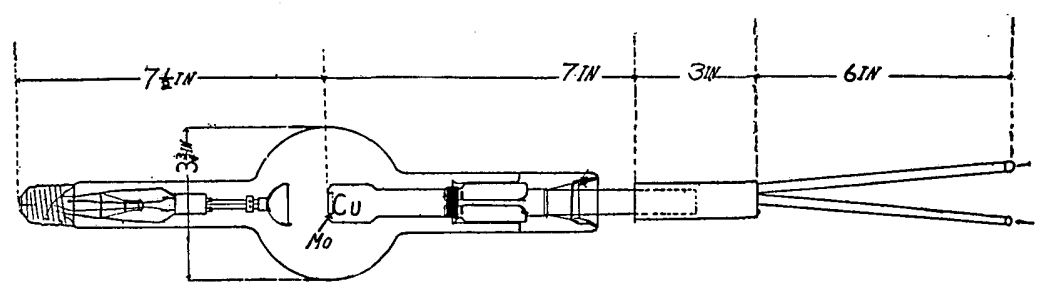

-Fig. 2. Diagram of water cooled Coolidge tube

The optimum D. C. voltage for the production of Mo $a$ is 28-30 kv, ${ }^{1}$ but operation on A. C. at $30 \mathrm{kv}_{\max }$ makes the time required for a satisfactory film so long that it is prohibitive for ordinary work. Experience shows that $30 \mathrm{kv}$ rMs is about the maximum voltage at which the "white" radiation may be easily filtered from the Mo characteristic rays. For this reason it was adopted as the standard operating voltage for this apparatus.

The Coolidge tube is operated directly from a transformer without any additional rectifying device. This necessitates a special design of transformer, for, although the secondary must supply power only on one half of the wave, the voltage of the inverse half-wave must not greatly exceed that of the useful half-wave. Otherwise there will be considerable trouble from corona during the inverse half-wave. The transformer has only one developed terminal, the other end of the secondary being grounded through the milliammeter. A protective spark-gap is provided across the milliammeter circuit so that the meter is always safe to handle even if one of its connections is broken. The filament of the Coolidge tube is heated by an extra coil at

${ }^{1}$ A. W. Hull, "A New Method of Crystal Analysis," Phys. Rev. 10, p. 661, 1917. 
the high-potential end of the secondary. Both ends of this coil, as well as the high-potential end of the secondary, are brought out through the high-potential terminal to a Kearsley stabilizer, ${ }^{2}$ which automatically lowers the filament temperature in case of excessive discharge currents through the tube. Fig. 3 gives a diagram of the connections. From the Kearsley stabilizer, the high-potential filament circuit goes directly to the cathode of the Coolidge tube which hangs directly above it. The anode end of the tube is in electrical contact with the table top, as described above, so that the circuit is completed by the grounded metal

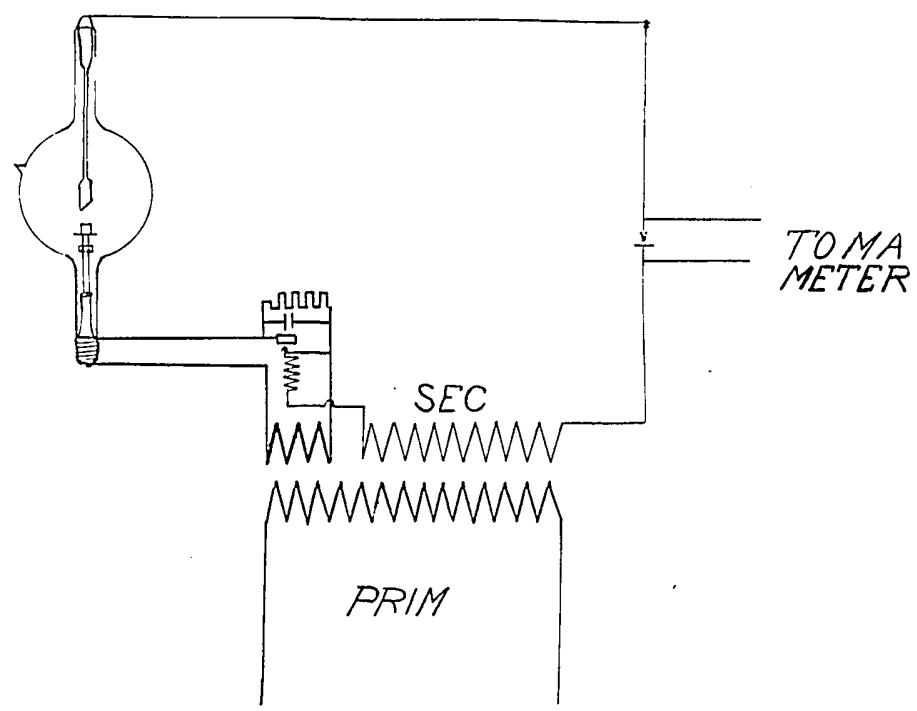

Fig. 3. Wiring diagram

table, and the milliammeter back to the transformer. In this way the high potential is entirely surrounded by grounded metal.

The switchboard contains the customary circuit breakers, operating switch, an auto-transformer for adjusting the primary voltage of the transformer, a voltmeter for reading the primary voltage, a milliammeter and a pressure-stat. This pressurestat is connected to the outlet end of the water cooled anode of

${ }^{2}$ W. J. Kearsley, Jr., "A New Type of Stabilizer for Use with the Coolidge Tube," Journal of Radiology, July, 1921. 
the Coolidge tube. In case the water pressure falls too low, the pressure-stat short-circuits the power line through a small resistance, thus tripping the circuit breakers and protecting the tube from being burned out. The water pressure at the inlet end of the tube should be not less than 25 nor more than $80 \mathrm{lb}$. per square inch. In setting up the apparatus, enough resistance to flow of water at the outlet is provided so that the pressure-stat opens the protective short-circuit when the inlet pressure is 25 lb. per square inch.

A small centrifugal blower, mounted alongside the transformer, provides an air blast against the cathode neck of the Coolidge tube. This prevents local heating of the glass by radiation from the cathode during continuous operation, which might otherwise evolve gas and thus destroy the space-charge characteristics of the tube, with consequent loss of the tube due to overload.

\section{The Slit System}

The slit system is suspended from a disk of $1 / 4$-inch plate glass which has a hole $61 / 4$ inches in diameter cut in the center. The system is composed of 15 radial sets of 3 slits each,- the first tw $>$ of which define the beam, while the third cuts off most of the secondary rays produced by the edges of the second slit. The whole slit system is formed on the surface of a cone whose basal angle is $5^{\circ}$. In this way each of the 15 slits utilizes the full projection of the focal spot of the Coolidge tube. The first slits are separated from each other by small brass partitions hung from the glass disk. The upper edges of these slits are formed by the edge of the glass disk itself. The lower edges are formed by the edges of small sectors of glass suspended between these partitions and the second slit. In this way the first slit is completely insulated from ground so as to avoid corona between it and the bulb of the Coolidge tube with consequent loss of the tube. If the first slit had not been insulated, it would have been necessary to have placed it further from the wall of the Coolidge tube, or to have inserted sheets of insulation (such as insulating paper or fiber) between the slit and the tube. In either case the time required to photograph the diffraction pattern would have been increased. 
The second and third slits are composed entirely of brass, which seems to be about as good as lead for the wave length employed. They are supported from the glass disk, but are electrically connected to the grounded cylinder of the table top by small set screws which prevent displacement of the slit system. The slit system is shown in Fig. 4.

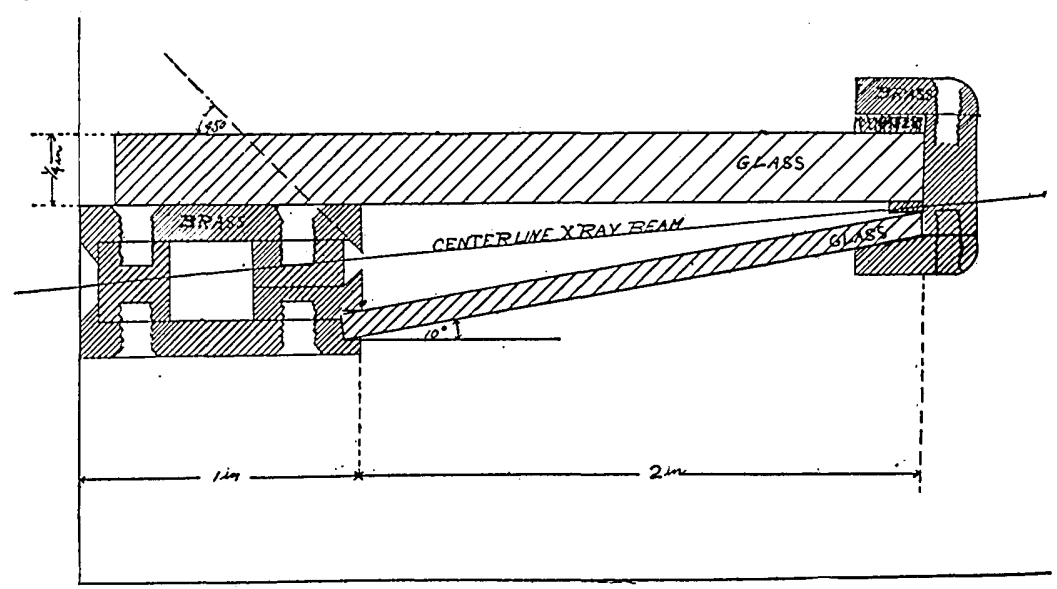

Fig. 4. Diagram of slit system

One of the filters mentioned above is fastened to the inner ring of slits, but is not shown in the diagram. Except for 15 rectangular spaces immediately in front of the slits, the whole surface of the filter is protected by nickel-plated brass from deterioration due to heat from the cathode reflected from the focal spot. At the slits, the white cardboard on which the filter is mounted is used for the same purpose.

The writer is well aware that numerous special types of slit systems have been devised by means of which X-Ray diffraction patterns may be photographed in less time than with the system described above. Although their merits for some highly specialized investigations are undoubted, they are hardly suited for general crystal research, nor for apparatus intended for most forms of routine factory-control work, either because of the large quantity of specimen required, or the cost of preparing the specimen in some special shape, or because certain lines in the pattern are enhanced at the expense of others. 
The Cassettes, Films, and Specimens

The cassettes (Fig. 5) serve not only as holders for the films, but also as holders for the specimens. The powdered specimens (preferably 200 mesh) may be mixed with pyroxylin and formed into thin, flat sheets, or they may be packed into thin-walled tubes of about 1/32 inch inside diameter, of paper, celluloid or special glass. The glass tubes are preferred for most substances because of the possibility of sealing the specimens so that they can not be affected by oxygen or moisture. Whether the specimen is prepared in the form of a flat sheet or packed in a tube, the specimenholder on the cassette automatically holds it in the path of the

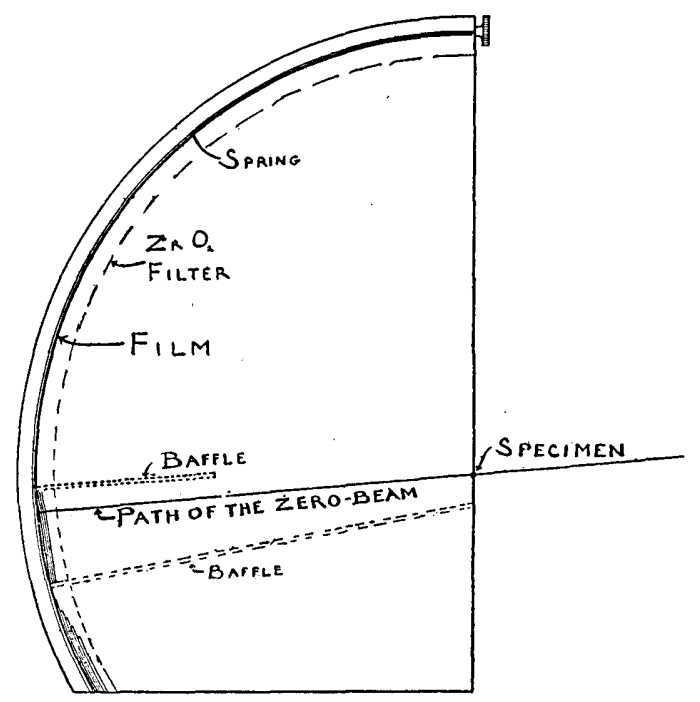

Fig. 5. Cassette

$\mathrm{X}$-Ray beam. In case the specimen contains elements of high atomic weight, it should be mixed with corn-starch or other amorphous substance, to decrease the capacity of the total mass.

A strip of thin, black celluloid is fastened to the cassette in the form of an arc, 8 inches in radius, with the specimen at the center. The photographic film is held against this by a wide flat brass spring which is drawn up tight by a screw. A light- 
baffle over the spring prevents the film from becoming lightstruck. The most satisfactory film for X-Ray diffraction work seems to be the Eastman "Dupli-tized X-Ray" film.

A "staircase" of copper is placed as an absorber in the path of the zero-beam. In this way there is always some part of the zero line on the film which has an exposure comparable to that of the first lines on the diffraction pattern. A molybdenum staircase is provided below the zero-beam for calibrating the photographic film.

A septum in the median plane of the cassette divides it into two symmetrical chambers, so that diffraction patterns of two substances may be taken on the same film for purposes of comparison. This is done by filling the glass specimen-tube half full of one substance, inserting a tiny plug of cotton, and then filling the remainder of the tube with the second substance. In work requiring the measurement of inter-planer distances with great accuracy it is recommended that one-half of the specimen-tube be filled with $\mathrm{NaCl}$. The theoretical spacings of $\mathrm{NaCl}$ (side of elementary cube $=2.810 \AA$ ) may then be used to give a correction curve for the film, thus avoiding errors due to any possible changes in the film itself.

A "range finder" is provided for adjusting the height of the Coolidge tube. It is built to fit the guides on the table top so that it may be slipped into place opposite any one of the 15 slits. A tungsten wire acts as the "specimen." A fluorescent screen is mounted at the outside end in a position corresponding to that of the film in the cassette. The screen is faced with glass as a partial $\mathrm{X}$-Ray protection to the operator. The Coolidge tube is at the proper height when the fluorescent screen shows that the shadow of the tungsten wire is in the center of the zero-line.

The Diffraction-Pattern

The photograph of the X-Ray diffraction pattern looks much like the familiar picture of a line spectrum. The diffraction pattern of $\mathrm{NaCl}$, taken with this apparatus, is shown in Fig. 6. A full account of the mathematical theory of the pattern is given elsewhere by A. W. Hull. ${ }^{3}$

${ }^{3}$ A. W. Hull, loc. cit. 
Using the classical equation for diffraction, $2 \mathrm{~d}=\mathrm{n} \lambda \sin \theta$

a scale has been calculated by which each line on the film may be directly interpreted in terms of the distance between the planes of atoms which produced the line. The apparatus has thus made a record of the diffraction pattern of the specimen and has enabled

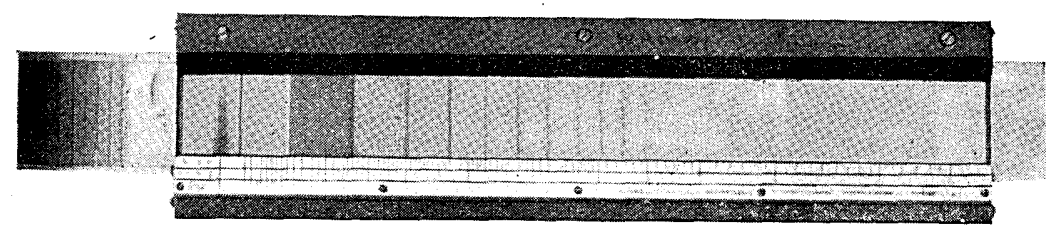

Fig. 6. Diffraction pattern of $\mathrm{NaCl}$ on scale, ready for measurement

the experimenter to record in Ångstrom units the spacings of all planes in the crystal which are more than . $55 \AA$ apart. Theoretically a diffraction pattern should be given by all planes having a spacing greater than $\lambda / 2(=.356 \AA)$, but except with very long exposure-time the lines from planes less than . $55 \AA$ are so faint as to be useless. Indeed, where a solution of the crystal structure can be found at all, it is usually possible to make a valid solution using only lines corresponding to planer distances of more than $.80 \AA$. When the doublet is resolved $\left(\lambda=.710 \AA, \lambda_{2}=.714 \AA\right)$ the readings of the two lines as given by the scale will be in the ratio of $1: 1.005$. In such a case the pattern is to be considered as showing a single line situated half-way between the two actual lines. The order of accuracy of readings of lines by means of the scale increases from the left hand end of the scale (large values) to the right hand end (small values). At $1.00 \AA$ it is of the order of 0.1 per cent. The limiting feature is the accuracy of the investigator in estimating the center of the line on the film. In case a solution of the crystal structure is found by the graphical method mentioned below, the final result is usually accurate to about $1 / 4$ per cent. It can then be checked by numerical calculation to about 0.2 per cent.

\section{USES}

The apparatus described above has two fields of usefulness,(1) in the identification of materials, (2) in the determination of crystal structure. 
(1) All solid metals and their alloys, and most solid compounds, are crystalline and show X-Ray diffraction patterns. Waxes at ordinary temperature, and glasses are amorphous and show no diffraction patterns. The diffraction pattern of a given substance, as measured quantitatively on the scale described above, is as characteristic of that substance as the density, solubility, meltingpoint or ability to form chemical compounds. As far as is now known, no two substances have identically the same diffraction pattern. At first it was thought that the patterns of Mo and W were identical, but Hull has since shown ${ }^{4}$ that the measurements of their diffraction patterns differ by about $1 / 4$ per cent. All other substances so far investigated which happen to have diffraction patterns of similar appearance are found, upon measurement with the scale described above, to differ from each other by several per cent. The apparatus therefore offers a convenient method for the qualitative analysis of crystaline substances. Any substance is completely identified when its pattern, measured on the scale, exactly matches the pattern of some substance whose identity is known. A large mass of such data is already available and will be published shortly in compact form.

This method of analysis has three advantages,-(a) only $.001 \mathrm{cc}$. of the specimen (ground to $200 \mathrm{mesh}$ ) is required; (b) the original sample is still available after the analysis is completed; (c) in a mixture of two compounds, the state of combination may be determined ${ }^{5}$-i.e.,

$$
\begin{aligned}
& \mathrm{NaF}+\mathrm{KCl} \text { can be distinguished from } \\
& \mathrm{NaCl}+\mathrm{KF}
\end{aligned}
$$

The ease with which substances may be identified should make the method useful as a means of laboratory control of factory products, especially in the case of metals and their alloys. The method of crystal analysis offers the most accurate method known for the determination of coefficient of expansion and density. In both cases a solution of the structure of the crystal is necessary, as described below.

${ }^{4}$ A. W. Hull. Physical Review, 17, p. 5, 1921.

${ }^{5}$ A. W. Hull, "A New Method of Chemical Analysis," Jour. Am. Chem. Soc. 41, p. 1168, 1919. 
(2) It is often desirable, not only in purely scientific investigations, but also in factory-control work, to determine the arrangement of atoms in the crystal. In case the crystal happens to belong to the cubic, hexagonal, or tetragonal systems (and most metals, alloys, halogen compounds, nitrates of monovalent metals, and carbonates of bivalent metals do); the crystal structure may be solved directly by the following method. ${ }^{6}$

The edge of a strip of paper is laid along the logarithmic scale of abscissae which is found at the bottom of each of the plots of Fig. 7, and the distance between planes in the crystal, as read off from the film in Angstrom units, is plotted on this edge. The strip of paper is now moved across the various plots keeping the edge always parallel to the axes of abscissae. When an exact match is found between the plot and the pattern marked on the edge of the paper then the crystal belongs to the system and subdivision marked on the plot, and the correct axial ratio is given by the intersection of the edge of the paper with the axis of ordinates. It is necessary that every line in the experimental pattern on the strip of paper be represented in the plot. If there is a single experimental line left over, the solution is not valid, no matter how - good a match is obtained with the rest of the lines. The only exception to this is when several lines left over in this way can be shown to all fit some other portion of one of the plots, thus indicating that two crystal forms are present. If the plot predicts lines which are not found in the experimental pattern it is necessary to show either that those lines ought, theoretically, to be too faint to be seen on the film, or that the crystal structure is more complicated than that for which the plot was made, so that certain lines disappear by interference. (Thus any pattern given on the triangular close-packing and rhombohedral plots will fit certain lines of the simple triangular plots at the same axial ratio, and all the patterns of the body-centered and face-centered tetragonal structures will similarly fit portions of the simple tetragonal plots.)

${ }^{6}$ For a more complete description of the method see Hull and Davey, "Graphical Determination of Hexagonal and Tetragonal Crystal Structures from X-Ray Data," Physical Review, 17, p. 5, 1921. 
Plots of the cubic system will be found at an axial ratio of 1.00 in the plots of the tetragonal system. ${ }^{7}$ The diamond division of the cubic system may be found by omitting the 2nd, 5 th, 8 th, 13 th, and 19th lines, counting from the right hand side of the plot, from the plot of the face-centered division of the cubic system.

After an exact match is found for the experimental pattern, it should be verified by computing the density of the specimen in the following manner,-

(a) If the match is in the cubic system, the density of the substance is given by

where

$$
\rho=\mathrm{n} \frac{\mathrm{M} \times 1.649 \times 10^{-24}}{\left(\mathrm{~d} \times 10^{-8}\right)^{3}}
$$

$\rho$ is the density

$\mathrm{M}$ is the molecular weight of the substance; $1.649 \times 10^{-24}$ is the mass in grams of one unit of molecular (or atomic) weight;

$\mathrm{d}$ is the side of the elementary cube,-(it is the distance in Ångstroms between the 100 planes in the crystal);

$\mathrm{n}$ is the number of points associated with a unit cube in the crystal lattice. $\mathrm{n}$ is

1 for the simple cubic lattice

2 for the body-centered cubic lattice

4 for the face-centered cubic lattice

8 for the diamond-cubic lattice.

If the line corresponding to the " 100 planes" is absent (as in the case of the body-centered and diamond divisions) then twice the distance corresponding to the second-order line, called in the plots " $100(2)-100$," must be used as the side of the elementary cube.

(b) If the match is found in the tetragonal system, the density is obviously

$$
\rho=\frac{\mathrm{M} \times 1.649 \times 10^{-24}}{c\left(\mathrm{~d} \times 10^{-8}\right)^{3}}
$$

where $c$ is the axial ratio, and $n$ has the same values as before.

(c) If the match is found in the hexagonal system, find the distance corresponding to the " 10.0 planes." (If the match is

7 They will also be found in the plots of the rhombohedral division of the hexagonal system. 


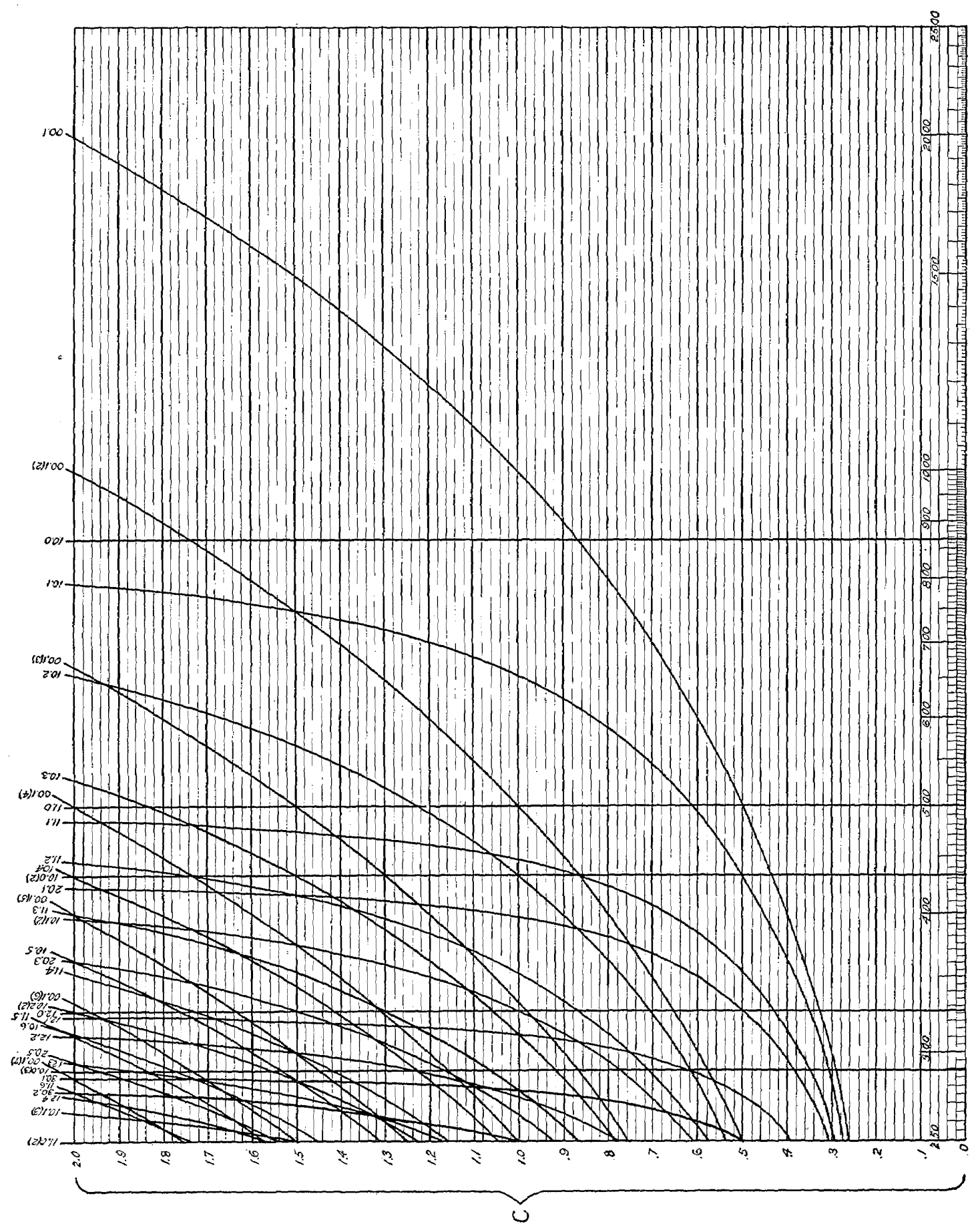




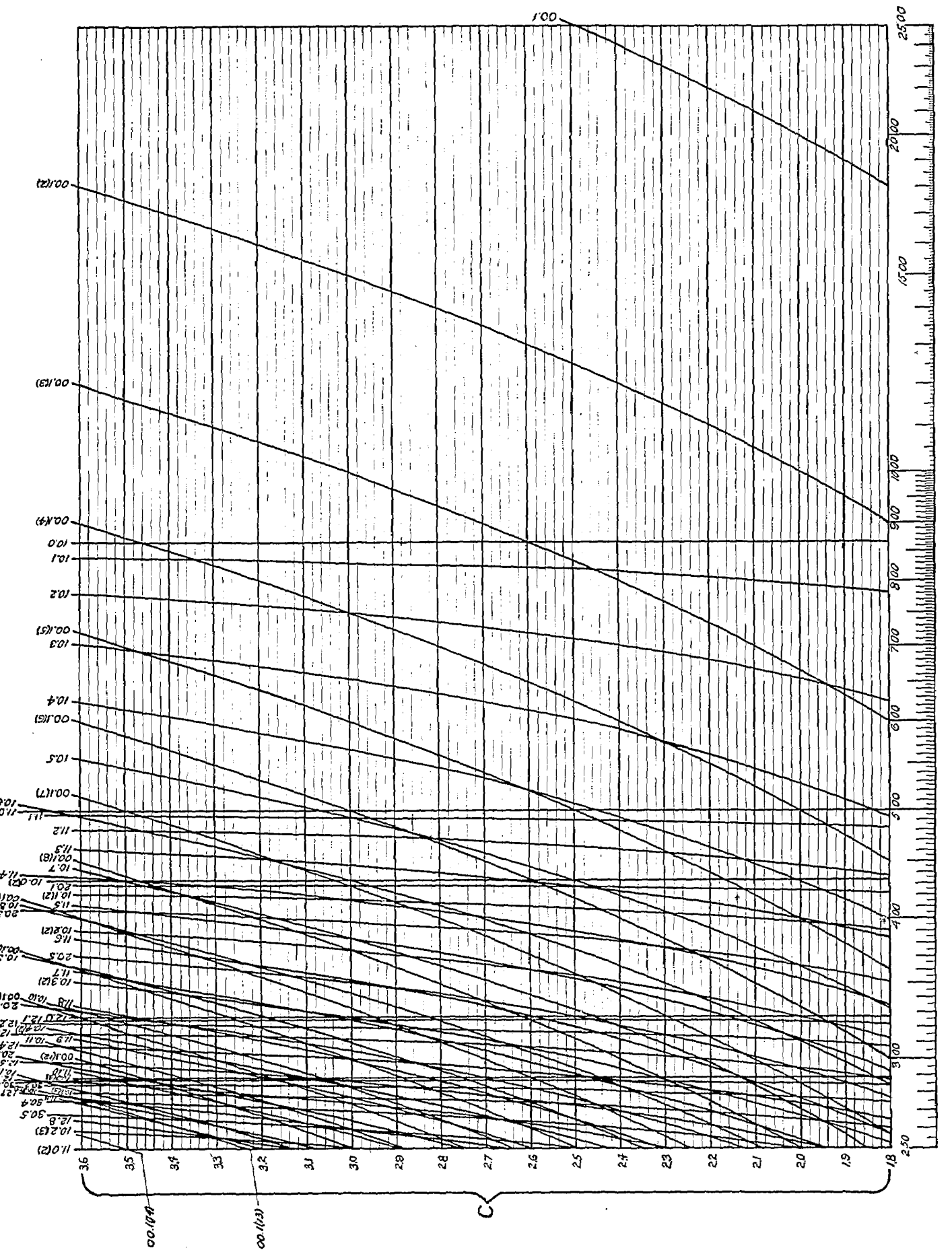




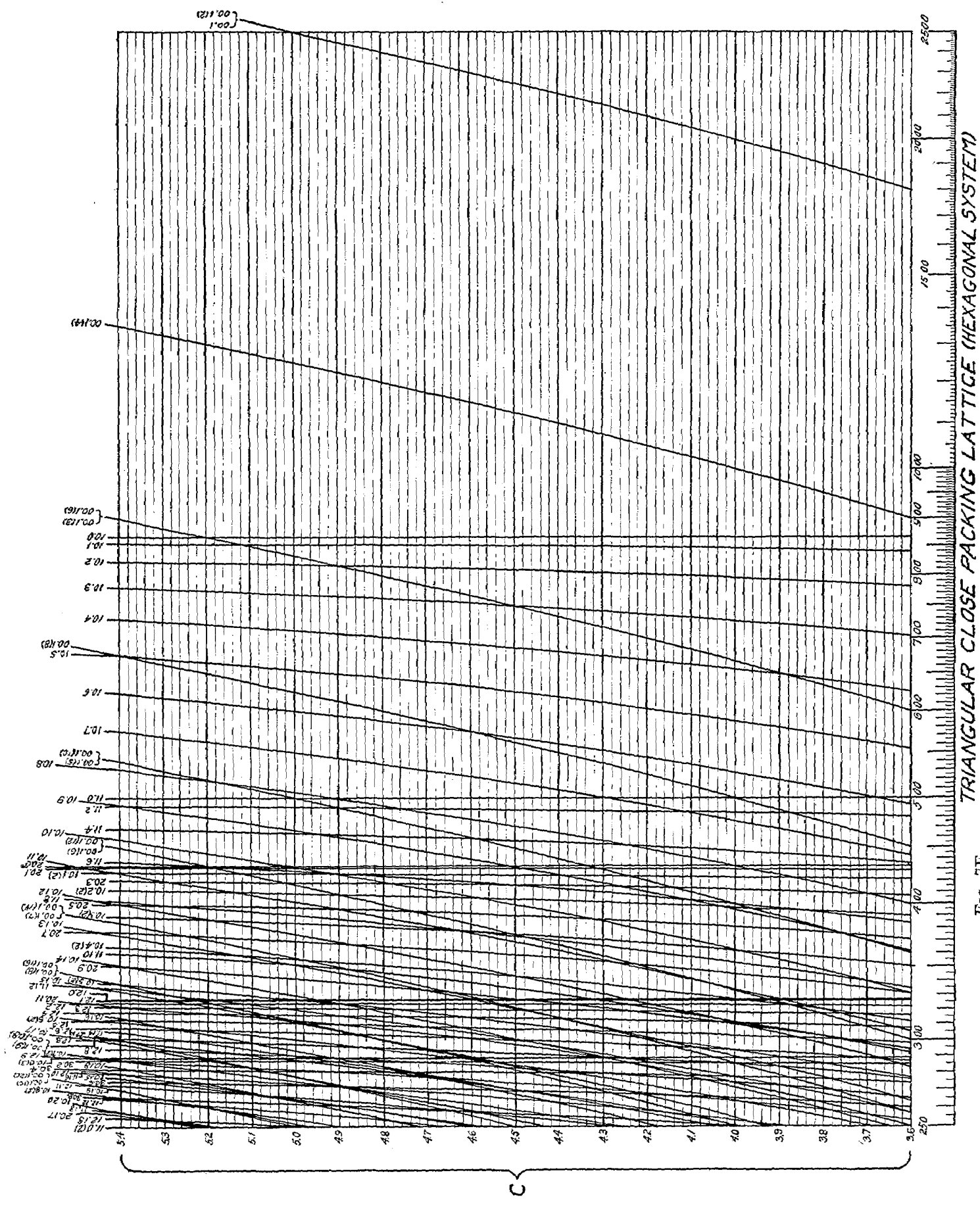




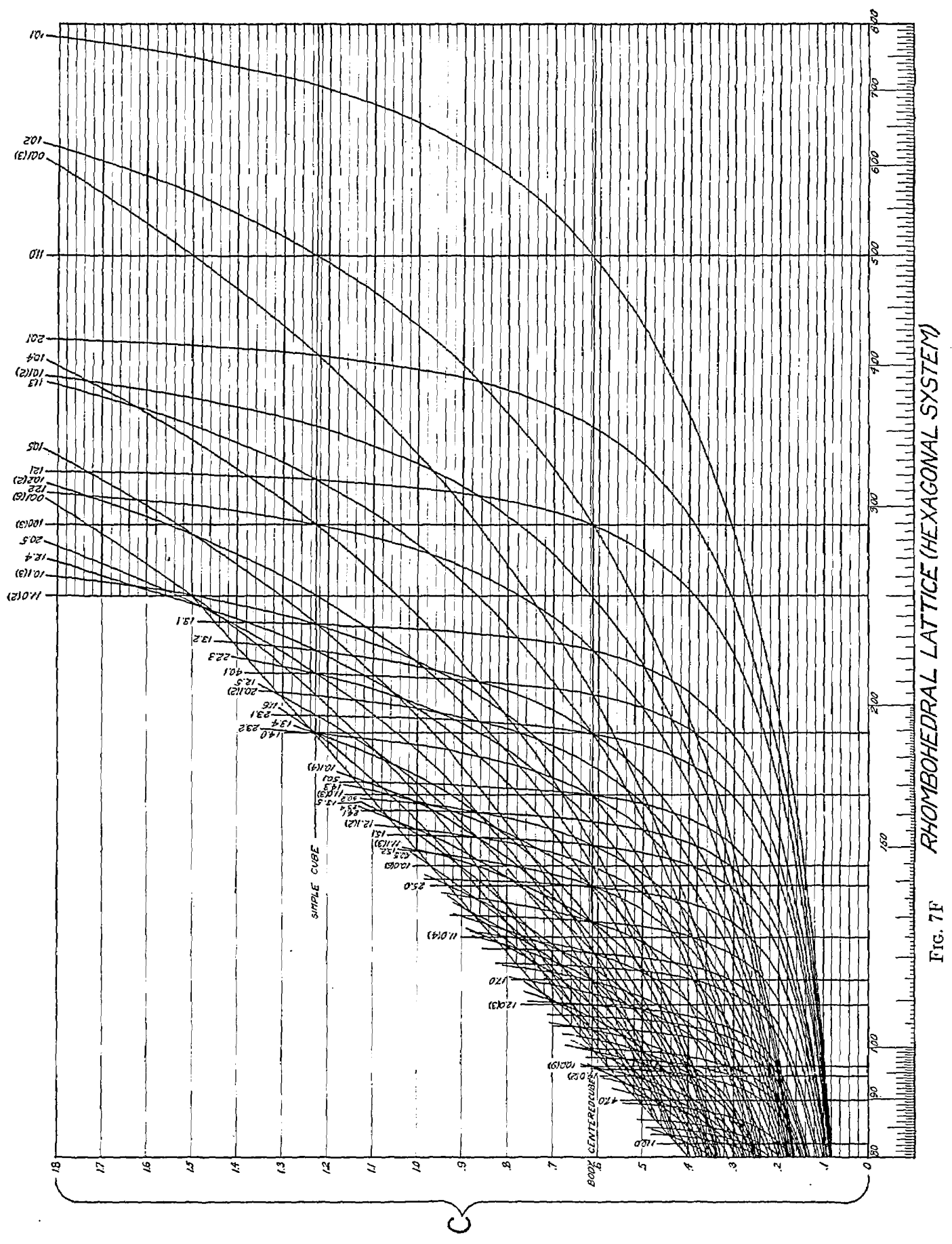




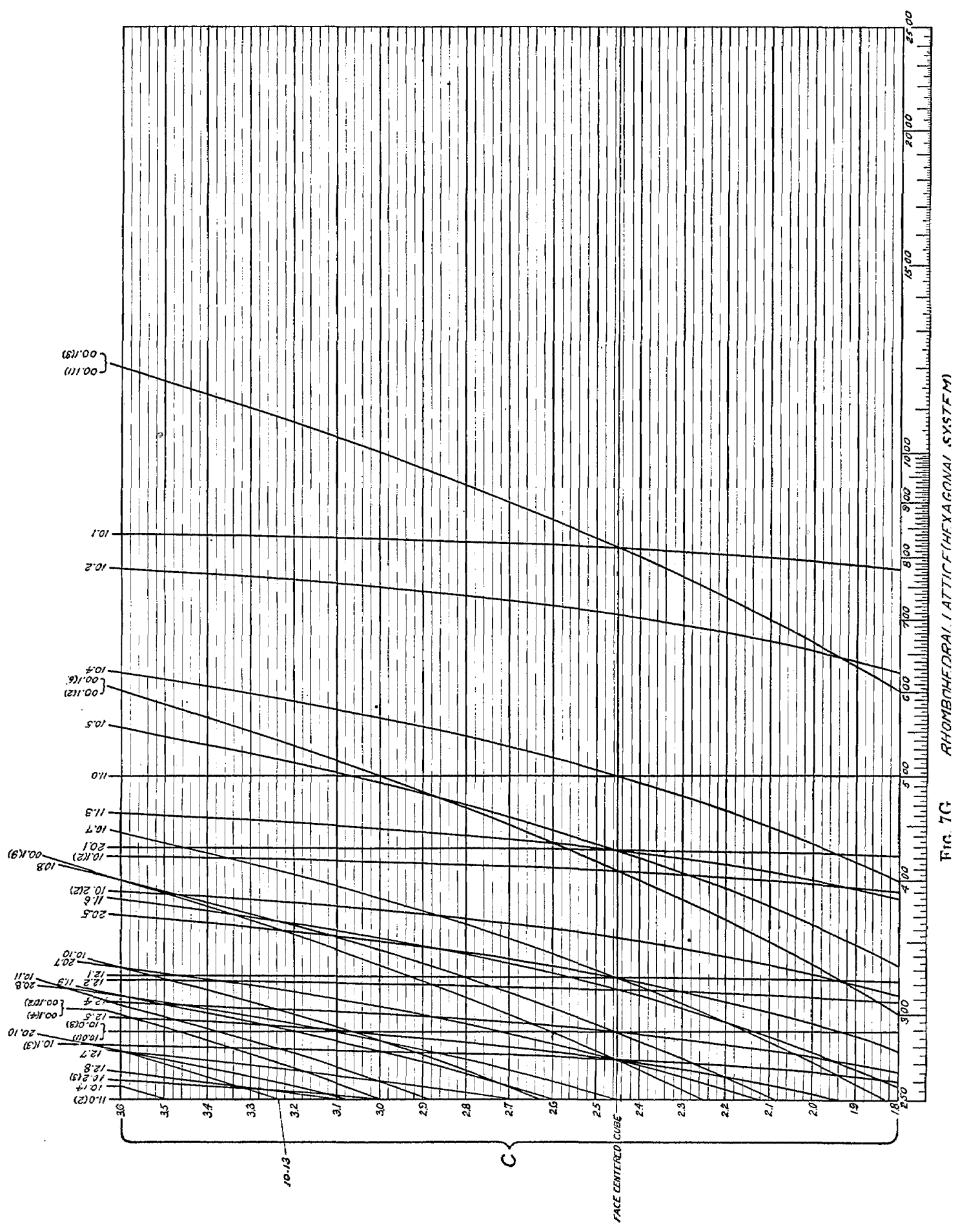









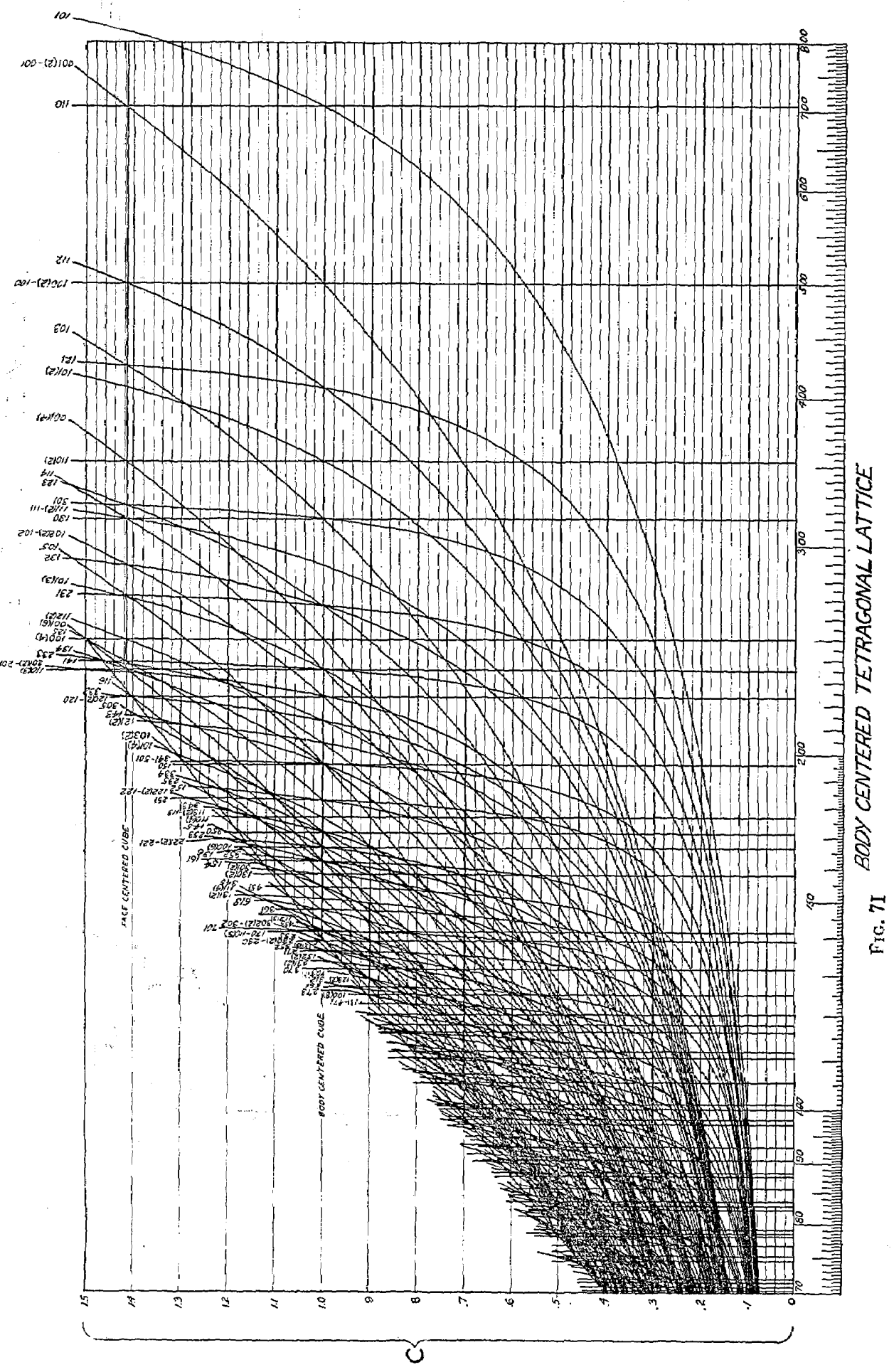




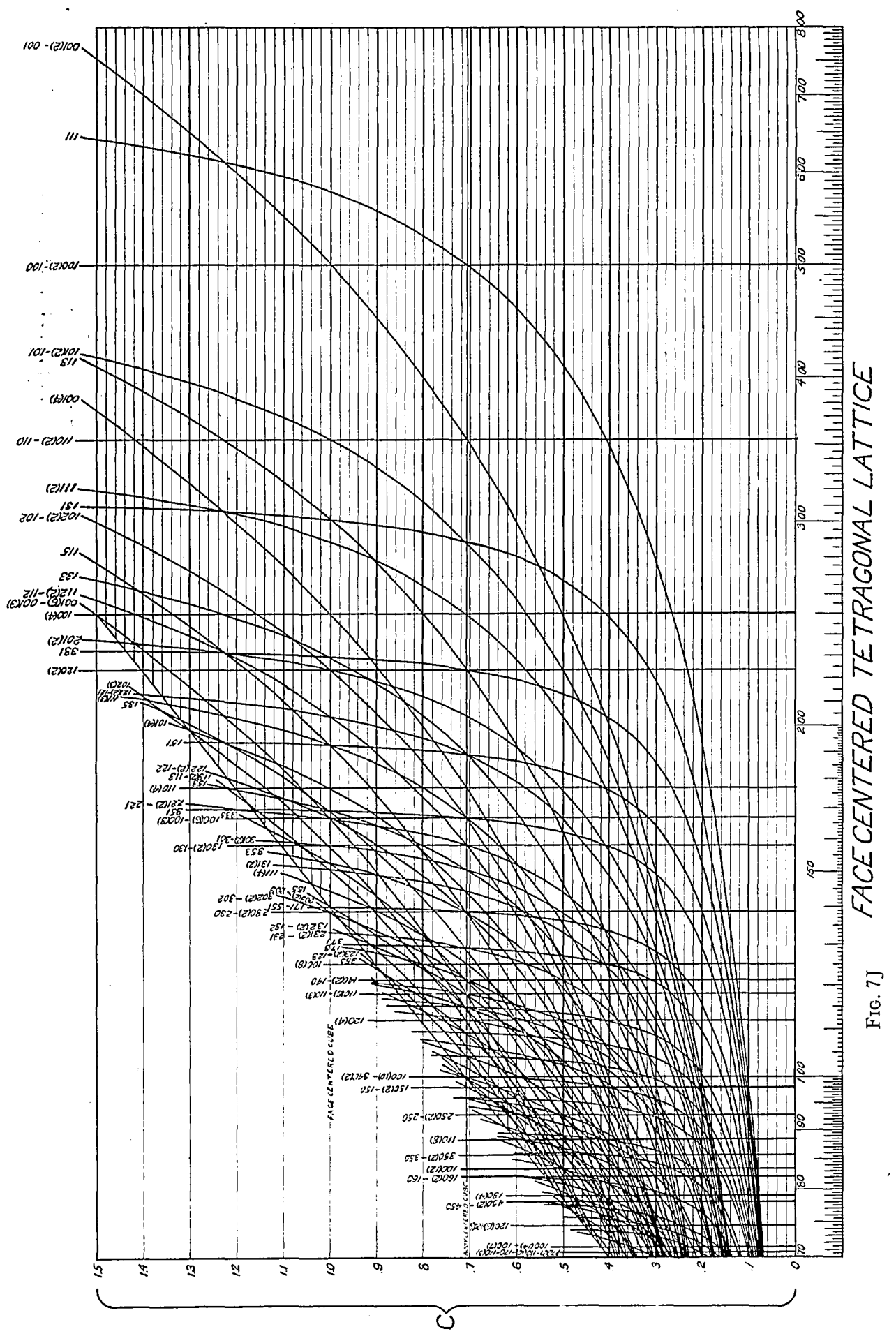


found in the rhombohedral division, three times the distance corresponding to the third-order line, called in the plots $\left\{\begin{array}{l}10.0(1) \\ 10.0(3)\end{array}\right.$ must be used.) This is the altitude of the equilateral triangle which forms the base of the unit prism. The side of the unit triangle is $2 / \sqrt{3}$ times this distance. The density of the specimen is therefore

$$
\rho=\mathrm{n} \frac{\mathrm{M} \times 1.649 \times 10^{-24}}{\sqrt{3 / 4} \mathrm{c}\left(\mathrm{a} \times 10^{-8}\right)^{3}}
$$

where $a$ is the side of the unit triangle.

$\mathrm{n}$ is $1 / 2$ for a simple triangular lattice

1 for a triangular close-packed lattice

$1 \frac{1}{2}$ for a rhombohedral lattice.

It is a pleasure to acknowledge the suggestions of A. W. Hull during the course of this work; also the assistance of E. O. Hoffman and H. A. Smith in the design of the slit system and the development of the water-cooled tube; R. Hergenrother in the laborious calculation and drawing of the plots; and Wm. F. Winter in the design and construction of the cassettes and the construction of the slit system.

RESEARCH. LABORATORY,

Generat Electric Co. 